\title{
Mechanism of Lactobacillus reuteri Probiotic in Increasing Intestinal Mucosal Immune System
}

\author{
Musjaya Guli ${ }^{1,2 \star}$, Sri Winarsih ${ }^{3}$, Wisnu Barlianto ${ }^{4}$, Oski Illiandri ${ }^{5}$, S. P. Sumarno ${ }^{3}$ \\ ${ }^{1}$ Department of Biology, Universitas Tadulako, Sulawesi Tengah, Indonesia; ${ }^{2}$ University of Brawijaya, Malang, Indonesia; \\ ${ }^{3}$ Department of Microbiology, School of Medicine, Universitas Brawijaya, Malang, Indonesia; ${ }^{4}$ Department of Pediatric, School \\ of Medicine, Brawijaya University, Malang, Indonesia; ${ }^{5}$ Department of Biomedicine, School of Medicine, Lambung Mangkurat \\ University, Banjarmasin, Indonesia
}

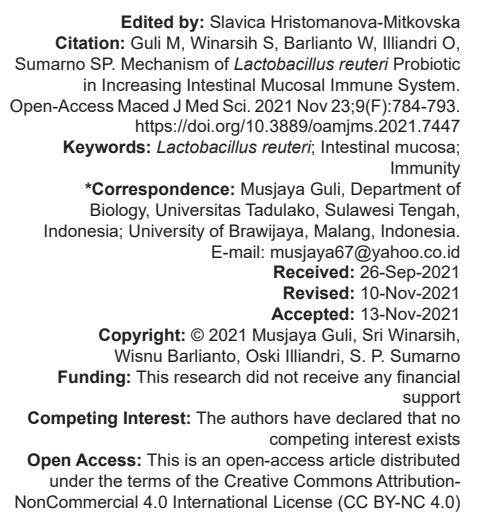

\begin{abstract}
Probiotics are defined as live microorganisms which, when consumed in adequate quantities as food ingredients provide health benefits to the host. Lactobacillus, Bifidobacterium, and Saccharomyces are three probiotics that are intensively used as probiotics in humans and animals. Probiotics have beneficial effects on health when given adequate amounts. The concept of probiotics on human health, is namely modulating the gut microbiota and its effect on the host. Probiotics play an important role in maintaining intestinal integrity through several different interactions including changes in cytokine expression in the mucosa. Probiotics compete with intestinal pathogens for mucosa receptors, thereby increasing interepithelial resistance. Probiotics were used as prophylaxis that could increase the expression of epithelial mucin, thereby reducing the translocation of pathogenic bacteria. The abnormal local immune response is characterized by decreased secretion of $\lg A$, thus allowing enterocyte attachment and local translocation of bacterial antigens, which are the main stimulation of pathological events. Colonic stasis can promote the growth of pathogenic bacteria which allow malignant porin bacterial strains to thrive. The gut microbiota have a major influence on human health. The microbial population has an important role in the host, such as the metabolic activity of probiotics producing energy and nutrient absorption, developing the host immune system, and preventing colonization and infection of pathogens. Lactobacillus reuteri is a heterofermentative bacterium that lives in the digestive tract of humans. L. reuteri has been used to treat infant necrotizing pseudomembrane. In this paper, the mechanism of $L$. reuteri to increase host immunological response will be reviewed.
\end{abstract}

\section{Introduction}

Probiotics are defined as live microorganisms which, when consumed in adequate quantities as food ingredients, provide health benefits to the host. Lactobacillus, Bifidobacterium, and Saccharomyces are three probiotics that are intensively used as probiotics in humans and animals [1]. Probiotics have beneficial effects on health when given adequate amounts. The concept of probiotics on human health, is namely modulating the gut microbiota and its effect on the host [2]. Probiotics play an important role in maintaining intestinal integrity through several interactions, including changes in mucosal cytokine expression. Probiotics compete with gut pathogens for mucosal receptors, thereby increasing the resistance between the epithelium [3]. Probiotics such as Lactobacillus casei sp. strain GG were used as prophylaxis that could increase epithelial mucin expression, thereby reducing the translocation of pathogenic bacteria. The abnormal local immune response is characterized by decreased secretion of $\lg \mathrm{A}$, thus allowing enterocyte attachment and local translocation of bacterial antigens, which are the main stimulation of pathological events. Colonic stasis can promote the growth of pathogenic bacteria which allow malignant porin bacterial strains to thrive. The gut microbiota have a major influence on human health. The microbial population has an important role in the host, such as the metabolic activity of probiotics to produce energy and nutrient absorption, the development of the host immune system, and the prevention of colonization and infection of pathogens.

Intestinal microbiota such as probiotics have a major influence on human health. Probiotic populations have an important role in the host, such as the metabolic activity of probiotics to produce energy and nutrient absorption, development of the host immune system, and prevention of colonization and infection of pathogens. Probiotics modify metabolism in the microbial ecosystem in the large intestine by increasing the production of short-chain fatty acids (SCFAs) such as acetate, propionic, and n-butyric acid. SCFA is the main product of carbohydrate breaking microbes and is the main anion in the large intestine. Propionic and n-butyric acid compress the colon to expel fluid. SCFA also plays a role in preparing energy for colonization and stimulating $\mathrm{Na}$ and water absorption from the large intestine, resulting 
in a decrease in diarrhea in the host [4]. Disturbances in the composition of the gut microbiota lead to the development of various pathologies. Manipulation of the gut microbiota through probiotics and other approaches is a therapeutic strategy to prevent or maintain the balance of the gut microbiota [5].

The intestinal ecosystem is characterized by reciprocal interaction between the microbiota, epithelium, and the mucosal immune system. This requires regulatory mechanisms and prevents aberrant responses to lead to pathological conditions. The epithelial layer is the first barrier against pathogens and is also the surface where the host and microbiota interact. Epithelial cells have an important role in sensing intestinal bacteria. Epithelial cells are equipped with various receptors to recognize specific molecular patterns on microbial pattern recognition receptors (PRRs). For example, a membrane binds to toll-like receptors (TLRs). TLR signals primarily through MyD88 activating key transcription factors such as nuclear factor kappa light chain B cells (NF-kB), triggering cytokine secretion, and activating host defense mechanisms [6]. Other PRR signals such as cytosolic nucleotide-binding oligomerization domain (NOD) receptors can modulate apoptosis and inflammatory responses [7]. The intestinal microbiota is not directly related to the intestinal epithelium physically but also is separated by a mucus layer. The commensal microbiota is on the outer layer, while the mucus layer is very dense on the inner layer to prevent pathogenic bacteria from adhesion to epithelial cells.

All bacteria secrete extracellular vesicles as a means of communication with the environment, characterized by the production of outer membrane vesicles (OMVs) by Gram-positive and Gram-negative bacteria. Vesicles act as pathways for secreting proteins and other components that are protected from the environment. Recent research on Gram-negative bacterial pathogens has shown that OMV is internalized in host cells and plays a role in virulence by releasing cytotoxic factors and mediators that can interfere with the host immune system [8]. At present, microbial vesicles play a key role in signaling processes in the intestinal mucosa [9].

OMV in commensal bacteria plays a role in promoting immunomodulatory effects and preventing colitis in rat experiments. This effect is mediated by capsule polysaccharide A (PSA) through TLR-2. However, transcriptomic analysis in dendritic cells (DCs) stimulated by PSA-OMV Bacteroides fragilis represents an independent PSA gene expression change [10]. Regarding Gram-positive bacteria, a study conducted using Bifidobacterium bifidum LMG13195, showed that this probiotic vesicle membrane can activate DC maturation that triggers regulatory $\mathrm{T}$-cell (Tregs) responses [11].

Sensing the gut microbiota by the host mucosal immune system plays a very important role in maintaining intestinal homeostasis and inducing a systemic protective response. Thus, manipulation of the gut microbiota is a potential alternative to maintain health and prevent disease. Probiotics are defined as live microorganisms which, when consumed in adequate amounts as food, provide health benefits to the host, for example; Lactobacillus, Bifidobacterium, and Saccharomyces are three probiotics that are intensively used as probiotics in humans and animals [12].

Several advantages of probiotics against the host defense system have been identified. One of the roles of probiotics is to block pathogenic bacteria by producing bactericidal substances and competing with pathogens for adhesion to the intestinal epithelium. For intestinal epithelial homeostasis, probiotics promote the survival of epithelial cells by enhancing barrier function and stimulating a protective response for intestinal epithelial cells. This can be done through enhancement of innate immunity and modulate inflammation through the TLR regulatory signaling pathway [13].

Metagenomic analysis broadens the understanding of probiotic genes involved in the regulation of the host immune response. Forty-two strains of Lactobacillus plantarum isolated from humans and the environment were evaluated for their capacity, for stimulation of interleukin (IL)-10 and IL-12 produced by peripheral blood mononuclear cells. Comparison of strain-specific cytokine responses and hybridization genome profiles using the WCFS1 L. plantarum DNA microarray. The gene is involved in encoding the $\mathrm{N}$-acetyl-glucosamine/galactosamine phosphotransferase system, the LamBDCA quorumsensing system, which is a component of the bacteriocin biosynthesis and transport pathway. Deletion of the WCFS1 gene in L. plantarum results in the loss of the capacity to stimulate cytokine production [14].

Several different genes of $L$. plantarum involved in the regulation of cytokine production by peripheral blood mononuclear have been identified, including six genes involved in the production or secretion of bacteriocins. One encodes bile salt hydrolase and the other encodes a transcriptional regulator [15]. Thus, it is shown that the regulation of responses by different immune cells is also present in certain probiotic genes. Genomic analysis of three closely related strains of Escherichia coli, respectively, strains 83,972 and Nissle 1917 were probiotic strains from the urinary tract and feces, and strain CF1073 was an uropathogen. The transcriptomic profile is the genomic profile of these three interrelated strains. The results showed that $E$. coli Nissle 1917 lived in urine, requiring three genes, yhak, yhcH, and ybi [16]. This indicates that the same functional gene profile has probiotic and pathogenic linkages. It is important to understand how these transcripts are similar but have different functions depending on their cellular relationships with each other.

Probiotic bacteria can induce a controlled host inflammatory response, including the release of inflammatory cytokines such as IL-6, IL-8, IL-1 
TNF-alpha, and TNF-beta IL-8 is involved in the migration of polymorphonuclear leukocytes across the intestinal barrier, toward the site of the lesion [17]. Lactobacillus strain probiotics can induce intestinal epithelial cells to increase the production of AMP and mucin, as the host's first line of defense against pathogens [18].

The role of probiotic Lactobacillus reuteri as immunomodulator has been widely accept, however, the mechanism of $L$. reuteri to increase host immunological response is still poorly understood. In this paper, the mechanism of $L$. reuteri as host immunomodulator will be reviewed.

\section{Probiotics and Mucin Production}

Probiotics can promote mucus secretion, as a mechanism to improve barrier function and pathogen exclusion. To support this concept, probiotics have been shown to increase mucin expression in vitro, play a role in barrier function and pathogen exclusion. Several species of Lactobacillus can increase mucin expression in Caco-2 (MUC2) and HT29 (MUC2 and 3 ) as the front line of intestinal cells, thus preventing pathogens from invading and adhering [19]. However, this effect depends on the adhesion of Lactobacillus to the epithelial cell layer which may not occur in vivo. The other group showed that Lactobacillus acidophilus A4 cell extract was sufficient to increase MUC2,3 and 5AC expression [20].

The in vivo studies mentioned above are inconsistent, there are still very few studies that have been carried out. Rats given VSL \#3 daily for 14 days showed no change in mucin expression or mucosal layer thickness [21]. In contrast, mice given daily VSL \#3 at the same dose for 7 days had a 60-fold increase in mucin expression as mucin secretion increased. MUC1 and MUC3 expression also increased with probiotic stimulation, but to a lesser extent [22]. Other studies have shown that probiotics are effective at preventing inflammation, as shown in animal studies [23]. Therefore, mucus production can be increased by probiotics in vivo, but in further studies, a conclusive statement is needed. Figure 1 shows the effect of the role of probiotics on the function of the intestinal epithelial barrier function.

\section{Probiotic Stimulation of Host Antimicrobial Peptide (AMP) Epithelial Cells}

There are two main families of intestinal AMPs; include defensins and cathelicidins. Both AMPs are

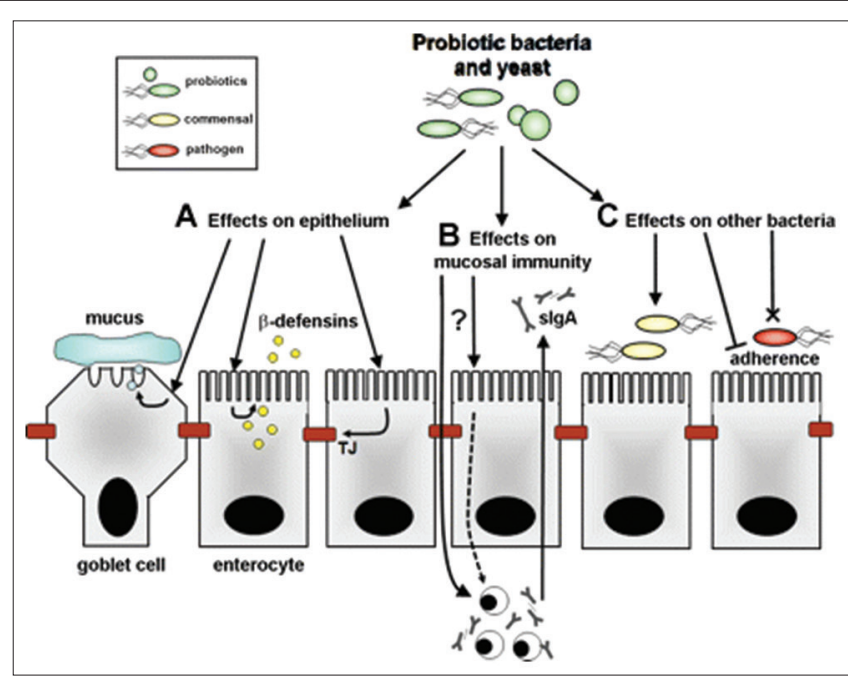

Figure 1: Effects of probiotic bacteria and yeast on intestinal epithelial barrier function. Various ways the role of probiotics on the epithelial barrier. Various multifactors to improve intestinal barrier function in the development and maintenance of homeostasis. Targets of probiotics against the epithelial barrier, including A. Direct role on the epithelium. Probiotics can increase the expression and secretion of mucin by goblet cells, thereby limiting the movement of bacteria in the mucosal layer. Expression and secretion of beta-defensin into mucus by epithelial cells to prevent commensal and pathogenic proliferation, thereby contributing to barrier integrity. Finally, probiotics can increase the stability of tight junctions, the epithelium decreases its permeability to pathogens and their products. B. Effects on mucosal immunity. Probiotics can increase the levels of cells produced in the lamina propria and promote the secretion of slgA into the luminal mucosal layer and limit colonization and binding by bacteria and their antigens, thereby contributing to intestinal colonization. Probiotics can alter the composition of the microbiota and/or gene expression, thus leading directly to the enhancement of the barrier through commensal bacteria. In addition, probiotics can directly kill or inhibit the growth of pathogenic bacteria through the expression of antimicrobials, such as bacteriocins. Probiotics can also compete with pathogens or commensals for binding sites on epithelial cell mucin, thereby preventing harmful colonization and contributing to barrier function [18]

cationic, expressed by gastrointestinal epithelial cells involved in host defense against pathogens. The only microbial or inflammatory stimulus for the induction of cathelicidin expression is butyrate, produced by the gut microflora. One study, using butyrate for the treatment of Shigella infection in rabbits, reported a significant reduction in dysentery, correlated with upregulation of cathelicidin. In subsequent studies, probiotics play a role in the regulation of cathelicidin in protecting the host from pathogenic infections.

In vitro studies demonstrated that the expression and secretion of HBD-2 were significantly in Caco- 2 cells stimulated by EcN, commensal E. coli strain DSM 17252 G2, several species of Lactobacillus or VSL\#3 [24]. The expression of hBD-1 and HD-6 was not altered by any of the probiotic bacteria. Furthermore, healthy humans who received Symbioflor 2 times for 3 weeks significantly increased HBD-2 protein [25]. In contrast, individuals tested with a placebo did not experience any changes. HBD-2 was still elevated after probiotic testing was discontinued, although to a lesser extent. Further studies of the host receptors involved 
are needed to understand the effect of probiotics on defensin production.

\section{Adhesion of Probiotics to Intestinal Epithelial Cells}

Probiotic bacteria also contribute to the intestinal barrier against invading pathogens in a way that is specific for binding sites to epithelial cells and the overlying mucosal layer. For example, Lactobacillus rhamnosus and $L$. acidophilus can adhere to intestinal epithelial cells. Free treatment of probiotic strains reduced the binding of enteropathogenic $E$. coli (EPEC). Furthermore, use with L. rhamnosus can inhibit the increase in EHEC induction. Furthermore, use with L. rhamnosus can inhibit the increase in EHEC induction, although this inhibitory effect is inhibited by the bacteria killer [26]. Another layer of protein from Lactobacillus can bind to epithelial cells so that there are no pathogenic bacteria on the surface of epithelial cells. The protein surface layer of Lactobacillus helveticus inhibited EHEC adhesion and increased permeability without altering pathogen growth. This is a function of the specific nature of the probiotic strain.

In vivo studies, probiotics prevent the adhesion of Citrobacter rodentium to intestinal epithelial cells by inhibiting the secretion of virulence factors involved in adhesion (EspB and Tir), not showing bactericidal activity. Giving probiotics to mice infected with attenuated diseases, including preventing epithelial damage and granulocyte infiltration. Hence, the inhibition of pathogen adhesion is a function of the probiotic mechanism in preventing intestinal infections [27].

\section{The Role of Probiotics in Inducing B Cells for IgA Production and Secretion in the Gut}

In the intestinal lamina propria, B cells differentiate into plasma cells and secrete dimeric $\lg \mathrm{A}$ antibodies. IgA polymer receptors on the basolateral surface of intestinal epithelial cells complex with IgA and transport to the apical cell surface, to be secreted into the intestinal lumen [28]. Mucosal immune responses to commensal bacteria including $\lg A$ production are independent of $\mathrm{T}$ lymphocytes and the organization of components of lymphoid tissue. Epithelial cells and DCs produce molecules such as ligand-inducing proliferation (APRIL), ligand DC40 and TGF $\beta$ which induce class switching $\lg A$ independent $T$ cells (van
Baarlen et al., 2007). Commensal bacteria such as L. plantarum WCFS1, Bacillus subsites JH642, and TLR-activated bacterial products induce intestinal epithelial cells to produce APRIL, triggering class switching of $\lg A$ to $\lg \mathrm{A} 2$. $\lg \mathrm{A} 2$ is an immunoglobulin that is common in the distal intestine and is more resistant to bacterial prostheses [29].

Mice are deficient in $\lg A$, as a result, their functional adaptive immune system is lacking. It upregulates genes related to innate immunity and downstream signaling pathways such as STATs and NFkB, observations of increased innate immunity [30]. Several studies have shown that probiotics stimulate plasma cells for IgA production. However, the ability to induce $\lg \mathrm{A}$ depends on the probiotic strain. One study giving Bifidobacterium lactis Bb-12 21 to volunteers for 21 days. The administration of this bacterial strain was positively correlated with an increase in the amount of fecal $\lg A$. Thus, administration of $B$. lactis can stimulate stimulation of $\lg A$ secretion in the intestine. Administration of $B$. bifidum $\mathrm{Bb}-11$ to mice showed that this probiotic strain increased the number of $\lg A$-secreting cells in the MLN and spleen, increasing systemically $\lg A$ in the gut. Another study, administration of fermented milk containing L. casei DN-114001, Lactobacillus delbrueckii subsp. bulgaricus, and Streptococcus thermophilus showed an increase in the number of $\lg A+$ cells in the intestine; small and large compared to Lactobacillus johnsonii NCC 533 and Lactobacillus paracasei NCC 2461. In disease-free mice, L. johnsonii NCC 533 and L. paracasei NCC 2461 can induce the formation of lymphoid follicles and increase the number of plasma IgA cells in the lamina propria [31].

The pattern of specific IgA elevation between L. johnsonii and L. paracasei, L. johnsonii significantly increased stimulation of specific IgA in Peyer's patches and intestinal lumen [32]. Meanwhile, L. paracasei resulted in a lower increase in specific IgA. The results of another study showed that the innate immune system plays an important role in the regulation of bacterial load. Differences between specific IgA production and bacterial load suggest that differences in immunogenicity may depend on the capacity of the strain for host defense.

Probiotics have been shown to play a role in increasing the production of $\lg \mathrm{A}$ in the host. For example, $L$. casei plays a very significant role in increasing cells for $\lg A$ production in the lamina propria of the small intestine. Peptides produced by Lactobacillus helveticus can increase the IgA response. However, not all probiotics have the same effect on slgA production. Mice that were given prebiotics (inulinenriched oligofructose) or symbiotic ( $L$. rhamnosus, Bifidobacterium, B. lactis, and inulin) played a role in increasing $\operatorname{sig} A$ production, indicating a diversity of stimuli in the gut. Furthermore, Saccharomyces boulardii can increase total slgA levels in conventional and model mice, indicating that there is an immune response carried out by probiotics against the host [33]. 
Commensal bacteria such as $L$. plantarum WCFS1, Bacillus subtilis JH642, and bacterial products that activate TLR can induce intestinal epithelial cells to produce APRIL, which triggers class switching of $\lg A$ to $\lg \mathrm{A} 2$, an immunoglobulin that is common in the distal intestine and is resistant to bacterial proteases in IgAdeficient mice. The commensal bacterium Bacteroides VPI-5482 can enhance innate immunity by increasing the activation of induced intratoxide synthetase and downstream signals such as NF-k $\beta$, upregulating genes associated with innate immunity [34].

Probiotics have many other immunomodulatory effects on the human gut, including promoting telogenic DC and regulatory T-cell phenotypes, inhibiting proinflammatory cytokine production, and increasing NK cell activity. It is shown in Figure 2 that showing the mechanism of probiotics in the digestive system.

. $\lg \mathrm{A}$ is resistant to proteases, is a complete function as a barrier function, and plays an important role in trapping pathogens/pathogenic material in the mucus layer through its ability to bind mucin. Probiotic strains such as Lactobacillus GG, B. lactis Bb-12, and Saccharomyces boulardii have been shown to increase $\lg$ A production and secretion through changes in the cytokine environment in the intestinal mucosa. Probiotic bacteria have been shown to induce the expression of TGF $\beta$, IL-10, and IL-6 in epithelial cells that have the potential to produce IgA through maturation and class switching of $B$ cells that support $\lg A$ [36]. Finally, probiotics can induce/increase the expression of polymer Ig receptors on the basolateral surface of intestinal epithelial cells to increase IgA transit through epithelial cells and into the intestinal lumen [37].

Oral administration of probiotics reaches the duodenum M cells to affect intraepithelial lymphocytes, acting as antigens that will stimulate mucosal plasma cells to secrete $\lg A$. The production of specific $\lg A$ triggers a response in the mesenteric lymph nodes to increase the number of IgA expression cells. The effect of increasing $\lg A$ in the intestine is to prevent local infection and absorption of allergens.

\section{Probiotic L. reuteri}

L. reuteri is a heterofermentative bacterium that lives in the digestive tract of humans and animals and is believed to be one of Lactobacillus species that

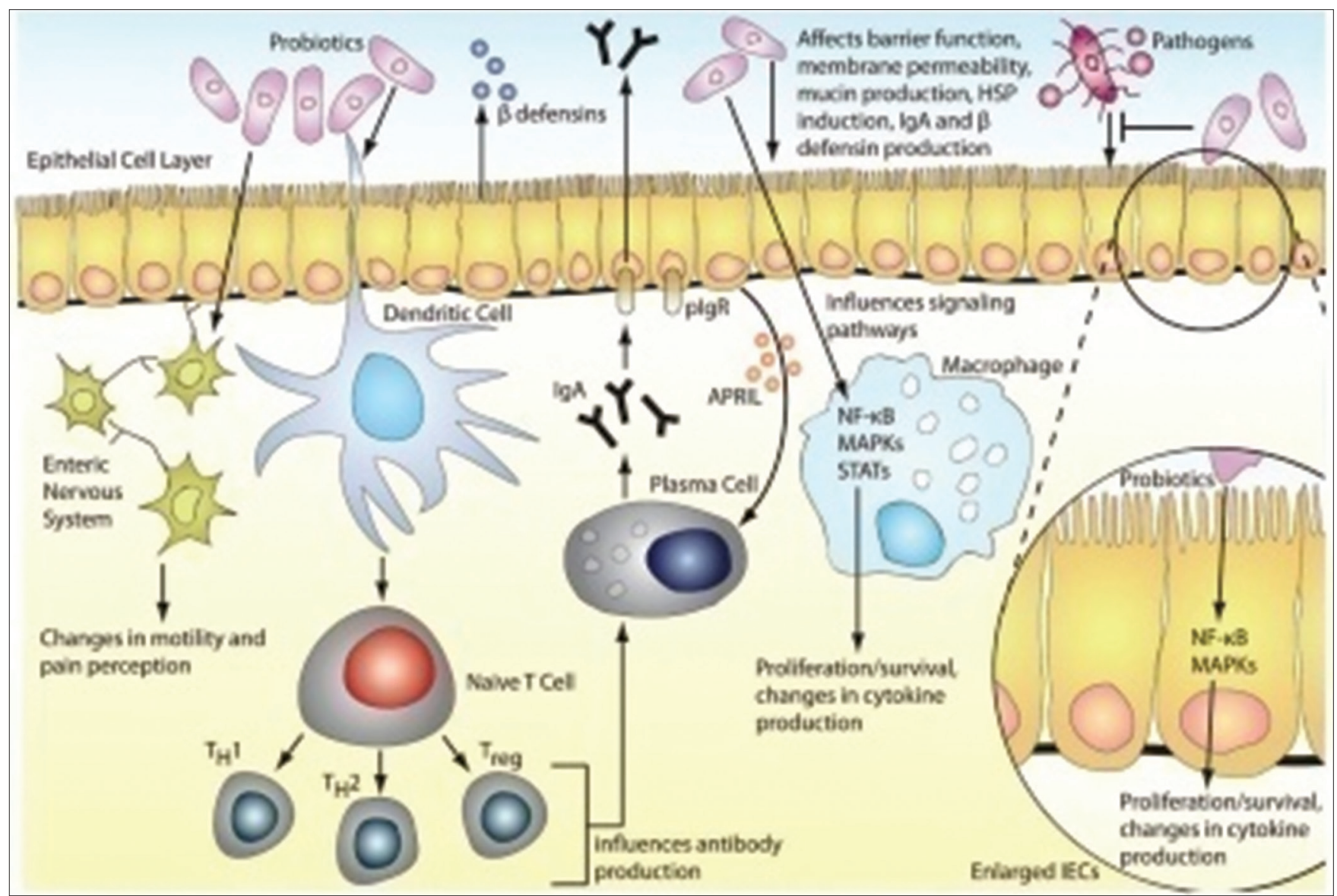

Figure 2: Mechanism of probiotics in the digestive system. Probiotics can manipulate microbial communities and suppress pathogen growth by inducing the production of beta-defensins and IgA. Probiotics can act as an intestinal barrier by maintaining tight junctions and inducing mucin production. Probiotics can modulate the production of cytokines through signaling pathways such as NF-kB and MAPK, thereby influencing the proliferation and differentiation of immune cells (such as $T$ cells) or epithelial cells. Intestinal motility can be modulated through regulation of receptor expression and secretion of neurotransmitters: APRIL, MAPK, NFkB, plgR, TSAT, and Tregs [35] 
came from humans. L. reuteri was first discovered in the 1980s, its strain was used as a probiotic [38]. $L$. reuteri is a species of bacteria whose main product is lactate from acid-producing bacteria. These bacteria are found in the human digestive tract in relatively small amounts. L. reuteri is also found in the intestines of other mammals and birds. Initially, L. reuteri has been used to treat necrotizing pseudomembrane, a disease characterized by gastrointestinal infection and inflammation that is harmful to infants, especially those born prematurely. L. reuteri has been used because of its anti-inflammatory effect. L. reuteri was first conducted in a study with the ATCC 55730 strain. In this study, $L$. reuteri was able to treat necrotizing pseudomembranes by administering oral supplements of Lactobacillus until the patient survived [39].

\section{Role of L. reuteri in Enhancing Host Immune Response}

L. reuteri is one of the probiotic species that live in the digestive tract (GIT) in vertebrates such as humans, pigs, and chickens. This is one of the probiotics that have been widely used as a probiotic in humans and animals [40]. This bacterium is a heterofermentative bacterium that is one of several Lactobacillus species that live in humans and animals. Several researchers isolated $L$. reuteri strain from human feces, breast milk, human vagina, human oral cavity, guinea pigs, pigs, and others. There is a lot of evidence showing that $L$. reuteri strain has probiotic characteristics that can provide host health benefits [40].

Administration of probiotic $L$. reuteri ATCC $6475\left(3.5 x^{10} 6\right.$ CFU) for 20-24 weeks, there was an increase in fur quality in female mice in the first 7 days. This was associated with an increase in the proliferation and activity of follicular sebocytes or skin dermal thickness by L. reuteri. ATCC 6475 strain did not appear to have decreased IL-10 cytokines in mice so that it can be used as an anti-inflammatory drug (Collins et al., 2013). Oral administration of L. reuteri ATCC 6475 can also affect the pro-inflammatory cytokine IL-17A in mice mediated by increased production of the anti-inflammatory cytokine IL-10 through the histamine activity of the $\mathrm{H} 2$ receptor. After binding to IL-10, IL-10 can suppress Th17 cells producing IL-17 [41].

Provision of L. reuteri DSM 17938 at a dose of $10^{6} \mathrm{CFU} / \mathrm{g}$ of body weight per day of the puppy with necrotizing enterocolitis (NEC) enhances $\mathrm{CD}^{+}$Treg cells $^{+} \mathrm{CD}^{+}$and $\mathrm{CD}^{+}{ }^{+}$Treg cells ${ }^{+}$the first few days (through food from their mothers) in the gut and spleen against controls [39]. This increase was associated with puppy survival. The increase in the population of Treg cells has also occurred in other populations, including normal mice given L. reuteri (ATCC 23272) $10^{9} \mathrm{CFU}$, there was an increase in Treg cells in the spleen tissue (60\% within 9 days) [42]. It may be that maintaining $T$-cell regulation through administration of $L$. reuteri as an anti-inflammatory effect is related to the reduction of pro-inflammatory cytokines (IL-6, TNF-alpha) and the increase of anti-inflammatory cytokines (IL-10).

Supplementation of $L$. reuteri DSM 17938 in healthy adults for 2 months $\left(5 \times 10^{8} \mathrm{CFU}\right)$ caused a significant increase in calprotectin in the fecal, as a sign of inflammation in the intestine. Although still within the normal range, the increase in calprotectin levels persisted at low levels for up to 6 months after discontinuation of $L$. reuteri supplementation. This dose is the same in people with cystic fibrosis (symptoms include intestinal inflammation, reducing calprotectin by $40 \%$ ). In hospitalized adults with diarrhea associated with antibiotics, supplementation of $\mathrm{L}$. reuteri ATCC 55730 at 108 CFU for 1 month can reduce the frequency of diarrhea from $50 \%$ to $7.7 \%$.

Commensal bacteria can well induce defensin-2 in epithelial cells, one of the commensal bacteria that can induce-defensin- 2 in oral epithelial cells. This means that the probiotic $L$. reuteri is a commensal bacterium that can act as an inducer to secrete defensin-2 which was detected in saliva samples. Oral administration of $L$. reuteri probiotics in the mouths of rats can increase defensin-2, which is expected to reduce the number of Streptococcus mutans bacteria thereby reducing dental caries in experimental rats. This occurs because the product of biologically active molecules from the surface of $L$. reuteri cell walls called MAMPs such as peptidoglycan (PG) and lipoteichoic acid (LTA) has the potential to activate surface receptors, namely, PRR from the host. PRRs such as TLR-2 and NOD-2 in the cytoplasm can then recognize several microbial components. PG and LTA are derived from the cell wall of $L$. reuteri which function as ligands for TLR2. The interaction of PG with LTA with TLR-2 and NOD-2 induces a signaling cascade involving NF-kB and inhibition of NF-kB kinase. PG and LTA from L. reuteri, then phosphorylation, ubiquitination, and degradation of NF-kB inhibitor protein (IKBK) occur, causing NF-kB to move to the nucleus, resulting in NF-kB activation, and activating the BD-2 promoter. Based on the results of the study, the administration of probiotic $L$. reuteri can increase the amount of BD-2 in the saliva of rats inoculated with S. mutans [43].

The results of the study explain how $L$. reuteri DSM 17938-CFS can be used as a probiotic. One study observed the response of DC types in regulating inflammation. LPS induces the expression of several genes, especially in monocyte-derived DC (Mo-DC). It also upregulated gene expression on Mo-DC more strongly than RA-DC. Thus, DCs play a role in the regulation of inflammation. Although $L$. reuteri-CFS only acts as LPS in inducing IL-10, IL-6, and IL-23 in both types of DC, $L$. reuteri CFS and LPS contribute 
to the regulation of various dendritic cell gene expressions [44].

$\mathrm{IL}-10$ is believed to be a key mediator in the regulatory environment. IL-10 deficiency can lead to the development of severe colitis pathology in the intestine. The results showed that $L$. reuteri DSM 17938 could increase IL-10 mRNA expression in mice. Meanwhile, in another study, IL-10 inhibited IL-23 production. However, IL-23 is also upregulated by RA-DC and Mo-DC after stimulation by $L$. reuteri. This contrasts with $L$. reuteri-mediated immunity that regulates IL-23, especially about the development of colitis. However, downstream IL-23 signals play a role in the production of IL-6 and IL-17 and play a role in the production of IL-22, as well as other cytokines from the IL-10 family. IL-22 in the intestine plays a role in maintaining the epithelial barrier and the induction of AMPs, such as defensins and lectins. In addition, IL-23 can reduce the inhibition of IL-12 production carried out by DCs, thereby indirectly controlling the overproduction of IFN- $\gamma$ by Th1 cells. This suggests that IL-23 also exerts an anti-inflammatory effect under certain circumstances.

IL-6 production increases after stimulation of $L$. reuteri, due to its pleiotropic activity, IL-6 can act as a pro-inflammatory but also plays a role in intestinal homeostasis. For example, it plays a major role in the production of $\lg A$ induced by the microbe $L$. reuteri and maintains the production of Th17 cells in the gut. Therefore, $L$. reuteri-CFS is induced to produce IL-6 cytokines to be able to influence the gut microenvironment, favoring a balance between a telogenic response to a harmless antigen, an effective response to infection.

L. reuteri CFS is induced by the production of several chemokines such as CXCL1, CXCL5, CCL3, CCL15, and CCL20 in RA-DC and Mo-DC which are involved in intestinal homeostasis. Local production of CXCL5 regulates neutrophil migration to the gut. CXCL5 in mice lacking neutrophils in the gut causes uncontrolled IL17 production. CCL5 has been shown to function as an AMP under stable conditions in the gut. CXCL1, apart from being an important component of the gut immune response, also plays a role in restoring mucosal barrier integrity in a mouse model. DCs secreting CCL3 and CCL20 may serve as an additional source in maintaining intestinal homeostasis.

Two genes are strongly influenced by $L$. reuteri in DC type, thrombospondin 1 (THBS1) and colonystimulating factor 2 (CSF2) [44]. Both factors are associated with anti-inflammatory activity. THBS1 is a negative autocrine that regulates DC activation by arresting cytokine production induced by microbial stimulation. CSF2 can trigger the production of regulatory molecules on DCs. There are important differences in the expression of surface markers between the two DC types associated with $L$. reuteri CFS exposure. L. reuteri increased the percentage of CD14+ cells in RA-DC, although these cells already had a higher percentage of CD14+ under unstimulated conditions. CD14 expression is believed to be related to the telogenic phenotype, conformance with RA $\mathrm{DC}$ regulation suggests that $L$. reuteri is capable of supporting this phenotype.

Probiotic bacteria can adapt to various stresses after ingestion by the host, including exposure to low $\mathrm{pH}$ in the stomach and contact with bile in the small intestine. L. reuteri strain had criteria including resistance to heat, low $\mathrm{pH}$, copper, and bile salts. Adhesion of probiotic strains to the host GIT is important for colonization and interaction with host cells to protect epithelial cells or modulate immunity. Several studies have shown that $L$. reuteri can colonize, can adhere to mucin and intestinal epithelial cells. L. reuteri 15007 exerts strong adhesion to caco- 2 cells, IEC-6 cells, IPEC-J2, and pork intestinal mucus. Mechanism of attachment and colonization involvement of $L$. reuteri is associated with mucus-binding protein, surface protein, D-alanyl-LTA, exopolysaccharide, and inulosucrase.

$L$. reuteri is reported to produce various antimicrobial substances such as lactic acid, hydrogen peroxide, reuterin, and reutericycline and has beneficial effects on the host. L. reuteri strain can inhibit the growth of enteric pathogens in vitro, including E. coli, Salmonella typhimurium, Staphylococcus epidermidis, Staphylococcus aureus, Helicobacter pylori, and rotavirus. In addition, $L$. reuteri also produces Vitamin B12 and can synthesize L-lysine and folic acid. Animal and human studies have shown that the oral administration of $L$. reuteri reduces the incidence and severity of diarrhea, prevents colic and NEC, maintains mucosal barrier function, and is immunomodulatory.

L. reuteri can stimulate or suppress the innate immune response through several mechanisms including modulation of pro-inflammatory cytokines. $L$. reuteri strains can be divided into two subsets, immunosuppressive (ATCCPTA 6475 and ATCCPTA 5289), and immunostimulating strains (ATCC 55730 and CF48-3A), each of which has therapeutic potential. Some paper reported administration of L. reuteri 15007 can enhance T-cell differentiation and induces ileal cytokine expression, suggesting that probiotic strains may modulate immune function. Hou showed that L. reuteri 15007 supplementation increased serumspecific anti-OVAlgG [40]. In neonatal piglets, L. reuteri was found to reduce ileal and IL-1 $\beta$ mRNA expression. Azevedo et al. (2012) found that $L$. reuteri combined with $L$. acidophilus can help to maintain immunologic homeostasis of rotavirus-infected neonates by regulating TGF- $\beta$ production [45].

$L$. reuteri, $L$. rhamnosus, and $L$. acidophilus were reported to reduce the duration of diarrhea in children. Colonization of $L$. acidophilus and $L$. reuteri can modulate the immune response to kill human rotavirus (HRV), affect the frequency of $B$ cells, monocytes/macrophages and DCs, TLRs, and innate cytokine expression patterns. L. reuteri and $L$. casei 
both have the potential to induce Treg cell development and are recognized by DCs. These interactions are important for priming regulator DCs. Lactobacillus complex consisting of Lactobacillus gasseri, L. reuteri, L. acidophilus, and Lactobacillus fermentum isolated from pigs was able to reduce $E$. coli bacteria in the intestine, thereby reducing the risk of diarrhea.

Oral administration of $L$. reuteri 100-23 cells in adult rats, in gastric colonization caused a mild inflammatory response in the ileal mucosa after an incubation period of 6 days. Transcription of genes encoding IL- $1 \alpha$ and IL- 6 is increased in small intestinal enterocytes. This is due to the maximum population development of Lactobacillus in the ileum. Although the number of Lactobacillus remained constant on day 21 after inoculation, at that time enterocyte, IL gene expression returned to its initial position. Therefore, the innate immune response to the presence of $L$. reuteri decreases the time of regulation, but the mechanism is unknown. Antibodies that react with proteins on the surface of $L$. reuteri 100-23 indicate an adaptive immune response, the presence of serum in mice is caused by bacterial colonization.

The combination of several probiotics such as L. acidophilus, L. casei, L. reuteri, B. bifidum, and $S$. thermophilus was stimulated by regulatory DCs to express IL-10, TGF- $\beta$, COX-2, and indoleamine 2-, 3-dioxygenase at high levels, which ultimately promotes $\mathrm{CD}^{+} \mathrm{Foxp}^{+}$Tregs from $\mathrm{CD} 4^{+} \mathrm{CD} 25^{-}$and increases the natural suppressing activity of $C D 4^{+} C D 25^{-}$Tregs. In addition, the probiotic mixture induced decreased $\mathrm{T}$ - and B-cell responses and downregulated Th1, Th2, and $\mathrm{TH} 17$ cytokines without inducing apoptosis. In vivo studies showed that the probiotic was suppressed by 2,4,6-trinitrobenzene sulfonic acid-induced intestinal inflammation. This is associated with an increase in $\mathrm{CD}^{+}{ }^{+}$Foxp $^{+}$Tregs at the site of inflammation. Thus, probiotics increase the generation of regulatory DCs to induce Tregs that play a role in potentially therapeutic therapies against inflammatory disorders.

\section{Conclusion}

Probiotic $L$. reuteri can induce intestinal inflammation-mediated- immunomodulation through both immunosuppressive and immunostimulant of pro-inflammation cytokine. This phenomenon can be done through modulation of AMPs in the intestine and pro-inflammatory cytokine. This AMP beta-defensin was released by local neutrophils which produce large amounts of the AMP calprotectin which plays a role in killing intestinal fungi and bacteria.

\section{References}

1. Fijan S. Microorganisms with claimed probiotic properties: An overview of recent literature. Int J Environ Res Public Health. 2014;11(5):4745-67. http://doi.org/10.3390/ijerph110504745 PMid:24859749

2. Shi LH, Balakrishnan K, Thiagarajah K, Ismail NI, Yin OS Beneficial properties of probiotics. Trop Life Sci Res. 2016;27(2):73-90. http://doi.org/10.21315/tlsr2016.27.2.6 PMid:27688852

3. Stavropoulou E, Bezirtzoglou E. Probiotics in medicine: A long debate. Front Immunol. 2020;11:2192. http://doi.org/10.3389/ fimmu.2020.02192

PMid:33072084

4. LeBlanc JG, Chain F, Martín R, Bermúdez-Humarán LG, Courau S, Langella P. Beneficial effects on host energy metabolism of short-chain fatty acids and vitamins produced by commensal and probiotic bacteria. Microb Cell Fact. 2017;16(1):79. http://doi.org/10.1186/s12934-017-0691-z PMid:28482838

5. Manor O, Dai CL, Kornilov SA, Smith B, Price ND, Lovejoy JC, et al. Health and disease markers correlate with gut microbiome composition across thousands of people. Nat Commun. 2020;11(1):5206. http://doi.org/10.1038/s41467-020-18871-1 PMid:33060586

6. Sugiyama K, Muroi M, Kinoshita M, Hamada O, Minai Y, SugitaKonishi $\mathrm{Y}$, et al. NF-кB activation via MyD88-dependent Tolllike receptor signaling is inhibited by trichothecene mycotoxin deoxynivalenol. J Toxicol Sci. 2016;41(2):273-9. http://doi. org $/ 10.2131 /$ jts. 41.273

PMid:26961612

7. Feerick CL, McKernan DP. Understanding the regulation of pattern recognition receptors in inflammatory diseases a "Nod" in the right direction. Immunology. 2017;150(3):237-47. http:// doi.org/10.1111/imm.12677 PMid:27706808

8. Schertzer JW, Whiteley M. Bacterial outer membrane vesicles in trafficking, communication and the host-pathogen interaction. J Mol Microbiol Biotechnol. 2013;23(1-2):118-30. http://doi. org/10.1159/000346770

PMid:23615200

9. Olsen I, Amano A. Outer membrane vesicles offensive weapons or good Samaritans? J Oral Microbiol. 2015;7:27468. http://doi. org/10.3402/jom.v7.27468

PMid:25840612

10. Shen Y, Torchia ML, Lawson GW, Karp CL, Ashwell JD, Mazmanian SK, et al. Outer membrane vesicles of a human commensal mediate immune regulation and disease protection. Cell Host Microbe. 2012;12(4):509-20. http://doi.org/10.1016/j. chom.2012.08.004

PMid:22999859

11. López P, González-Rodríguez I, Sánchez B, Gueimonde M Margolles A, Suárez A. Treg-inducing membrane vesicles from Bifidobacterium bifidum LMG13195 as potential adjuvants in immunotherapy. Vaccine. 2012;30(5):825-9. http://doi. org/10.1016/j.vaccine.2011.11.115 PMid:22172507

12. Yan F, Polk DB. Probiotics and immune health. Curr Opin Gastroenterol. 2011;27(6):496-501. http://doi.org/10.1097/ MOG.0b013e32834baa4d PMid:21897224

13. Vanderpool C, Yan F, Polk DB. Mechanisms of probiotic action Implications for therapeutic applications in inflammatory bowel 
diseases. Inflamm Bowel Dis. 2008;14(11):1585-96. http://doi. org/10.1002/ibd.20525

PMid:18623173

14. van den Nieuwboer M, van Hemert S, Claassen E, de Vos WM Lactobacillus plantarum WCFS1 and its host interaction: A dozen years after the genome. Microb Biotechnol. 2016;9(4):452-65. http://doi.org/10.1111/1751-7915.12368 PMid:27231133

15. Meijerink $M$, van Hemert $S$, Taverne $N$, Wels $M$, de Vos $P$, Bron PA, et al. Identification of genetic loci in Lactobacillus plantarum that modulate the immune response of dendritic cells using comparative genome hybridization. PLOS One. 2010;5(5):e10632. http://doi.org/10.1371/journal.pone.0010632 PMid:20498715

16. Hancock V, Dahl M, Klemm P. Probiotic Escherichia coli strain Nissle 1917 outcompetes intestinal pathogens during biofilm formation. J Med Microbiol. 2010;59(Pt 4):392-9. http://doi. org/10.1099/jmm.0.008672-0

\section{PMid:20110388}

17. Hofman PM. Pathobiology of the neutrophil-intestinal epithelia cell interaction: Role in carcinogenesis. World J Gastroenterol. 2010;16(46):5790-800. http://doi.org/10.3748/wjg.v16.i46.5790 PMid:21154999

18. Ohland CL, Macnaughton WK. Probiotic bacteria and intestinal epithelial barrier function. Am J Physiol Gastrointest Liver Physiol. 2010;298(6):G807-19. http://doi.org/10.1152/ ajpgi.00243.2009 PMid:20299599

19. Mack DR, Ahrne S, Hyde L, Wei S, Hollingsworth MA Extracellular MUC3 mucin secretion follows adherence of Lactobacillus strains to intestinal epithelial cells in vitro. Gut, 2003;52(6):827-33. http://doi.org/10.1136/gut.52.6.827 PMid:12740338

20. Otte JM, Podolsky DK. Functional modulation of enterocytes by gram-positive and gram-negative microorganisms. Am J Physiol Gastrointest Liver Physiol. 2004;286(4):G613-26. http://doi. org/10.1152/ajpgi.00341.2003 PMid: 15010363

21. Gaudier E, Michel C, Segain JP, Cherbut C, Hoebler C. The VSL\# 3 probiotic mixture modifies microflora but does not heal chronic dextran-sodium sulfate-induced colitis or reinforce the mucus barrier in mice. J Nutr. 2005;135(12):2753-61. http://doi. org/10.1093/jn/135.12.2753

PMid: 16317116

22. Caballero-Franco C, Keller K, De Simone C, Chadee K. The VSL\#3 probiotic formula induces mucin gene expression and secretion in colonic epithelial cells. Am J Physiol Gastrointest Liver Physiol. 2007;292(1):G315-22. http://doi.org/10.1152/ ajpgi.00265.2006

PMid:16973917

23. Boirivant $M$, Strober $W$. The mechanism of action of probiotics. Curr Opin Gastroenterol. 2007;23(6):679-92. http://doi. org/10.1097/MOG.0b013e3282f0cffc PMid:17906447

24. Beimfohr C. A review of research conducted with probiotic $E$. coli marketed as symbioflor. Int J Bacteriol. 2016;2016:3535621. http://doi.org/10.1155/2016/3535621

PMid:27995179

25. Simon E, Călinoiu LF, Mitrea L, Vodnar DC. Probiotics, prebiotics, and synbiotics: Implications and beneficial effects against irritable bowel syndrome. Nutrients. 2021;13(6):2112. http://doi.org/10.3390/nu13062112 PMid:34203002

26. Li N, Pang B, Li J, Liu G, Xu X, Shao D, et al. Mechanisms for Lactobacillus rhamnosus treatment of intestinal infection by drug-resistant Escherichia coli. Food Funct. 2020;11(5):4428-45. http://doi.org/10.1039/d0fo00128g

PMid:32374342

27. Monteagudo-MeraA, Rastall RA, Gibson GR, Charalampopoulos D, Chatzifragkou $A$, et al. Adhesion mechanisms mediated by probiotics and prebiotics and their potential impact on human health. Appl Microbiol Biotechnol. 2019;103(16):6463-72. http:// doi.org/10.1007/s00253-019-09978-7

PMid:31267231

28. Wells JM, Mercenier A. Mucosal delivery of therapeutic and prophylactic molecules using lactic acid bacteria. Nat Rev Microbiol. 2008;6(5):349-62. http://doi.org/10.1038/nrmicro1840 PMid:18345021

29. van Baarlen $P$, van Belkum $A$, Summerbell $R C$, Crous $P W$ Thomma BP. Molecular mechanisms of pathogenicity: How do pathogenic microorganisms develop cross-kingdom host jumps? FEMS Microbiol Rev. 2007;31(3):239-77. http://doi. org/10.1111/j.1574-6976.2007.00065.x

PMid: 17326816

30. Dorrington MG, Fraser ID. NF-KB signaling in macrophages: Dynamics, crosstalk, and signal integration. Front Immunol. 2019;10:705.

PMid:31024544

31. Galdeano CM, Cazorla SI, Dumit JM, Vélez E, Perdigón G. Beneficial effects of probiotic consumption on the immune system. Ann Nutr Metab. 2019;74(2):115-24. http://doi. org/10.1159/000496426

PMid:30673668

32. Hara S, Sasaki T, Satoh-Takayama N, Kanaya T, Kato T, Takikawa $\mathrm{Y}$, et al. Dietary antigens induce germinal center responses in Peyer's patches and antigen-specific IgA production. Front Immunol. 2019;10:2432. http://doi. org/10.3389/fimmu.2019.02432

PMid:31681315

33. Qamar A, Aboudola S, Warny M, Michetti P, Pothoulakis C LaMont JT, et al. Saccharomyces boulardii stimulates intestinal immunoglobulin a immune response to Clostridium difficile toxin A in mice. Infect Immun. 2001;69(4):2762-5. http://doi. org/10.1128/IAl.69.4.2762-2765.2001 PMid: 11254650

34. Hemarajata P, Versalovic J. Effects of probiotics on gut microbiota: Mechanisms of intestinal immunomodulation and neuromodulation. Ther Adv Gastroenterol. 2013;6(1):39-51. http://doi.org/10.1177/1756283X12459294

PMid:23320049

35. Thomas CM, Versalovic J. Probiotics-host communication Modulation of signaling pathways in the intestine. Gut Microbes. 2010;1(3):148-63. http://doi.org/10.4161/gmic.1.3.11712 PMid:20672012

36. Hu R, Lin H, Wang M, Zhao Y, Liu H, Min Y, et al. Lactobacillus reuteri-derived extracellular vesicles maintain intestinal immune homeostasis against lipopolysaccharide-induced inflammatory responses in broilers. J Anim Sci Biotechnol. 2021;12(1):25.

37. Reséndiz-Albor AA, Reina-Garfias $H$, Rojas-Hernández $S$, Jarillo-Luna A, Rivera-Aguilar V, Miliar-García A, et al. Regionalization of plgR expression in the mucosa of mouse small intestine. Immunol Lett. 2010;128(1):59-67. http://doi. org/10.1016/j.imlet.2009.11.005 PMid:19925828

38. Casas I, Dobrogosz W. Validation of the probiotic concept Lactobacillus reuteri confers broad-spectrum protection against disease in humans and animals. Microbial Ecol Health Dis. 2000;12:247-85. https://doi.org/10.1080/08910600050216246-1

39. Hoang TK, He B, Wang R, Tran DQ, Rhoads JM, Liu Y. Protective effect of Lactobacillus reuteri DSM 17938 against experimental 
necrotizing enterocolitis is mediated by Toll-like receptor 2 . Am J Physiol Gastrointest Liver Physiol. 2018;315(2):G231-40. https://doi.org/10.1152/ajpgi.00084.2017

\section{PMid:29648878}

40. Hou C, Zeng X, Yang F, Liu H, Qiao S. Study and use of the probiotic Lactobacillus reuteri in pigs: A review. J Anim Sci Biotechnol. 2015;6(1):14. https://doi.org/10.1186/ s40104-015-0014-3

PMid:25954504

41. Collins FL, Rios-Arce ND, Schepper JD, Jones AD, Schaefer L, Britton RA, et al. Beneficial effects of Lactobacillus reuteri 6475 on bone density in male mice is dependent on lymphocytes. Sci Rep. 2019;9(1):14708. https://doi.org/10.1038/ s41598-019-51293-8 PMid:31605025

42. Liu Y, Tran DQ, Fatheree NY, Rhoads JM. Lactobacillus reuteri DSM 17938 differentially modulates effector memory $\mathrm{T}$ cells and Foxp3+ regulatory $\mathrm{T}$ cells in a mouse model of necrotizing enterocolitis. Am J Physiol Gastrointest Liver Physiol. 2014;307(2):G177-86. https://doi.org/10.1152/ ajpgi.00038.2014

PMid:24852566

43. Vestman NR, Hasslöf P, Keller MK, Granström E, Roos S Twetman S, et al. Lactobacillus reuteri influences regrowth of mutans streptococci after full-mouth disinfection: A double-blind, randomised controlled trial. Caries Res. 2013;47(4):338-45. https://doi.org/10.1159/000347233

PMid:23486236

44. Haileselassie Y, Navis M, Vu N, Qazi KR, Rethi B, SverremarkEkström E. Postbiotic modulation of retinoic acid imprinted mucosal-like dendritic cells by probiotic Lactobacillus reuteri 17938 in vitro. Front Immunol. 2016;7:96. https://doi. org/10.3389/fimmu.2016.00096

PMid:27014275

45. Azevedo MS, Zhang W, Wen K, Gonzalez AM, Saif LJ, Yousef $A E$, et al. Lactobacillus acidophilus and Lactobacillus reuteri modulate cytokine responses in gnotobiotic pigs infected with human rotavirus. Benef Microbes. 2012;3(1):33-42.

PMid:22348907

Author Query???

AQ6: Please check the figures 1 and 2 caption

AQ1: Kindly provide updated image 